\title{
Contribution of nonlinearity in tsunami generated by submarine earthquake
}

\author{
M. A. Nosov, S. V. Kolesov, and A. V. Denisova \\ M. V. Lomonosov Moscow State University, Faculty of Physics, Moscow, Russia \\ Received: 16 June 2007 - Revised: 10 October 2007 - Accepted: 12 October 2007 - Published: 2 January 2008
}

\begin{abstract}
Rapid co-seismic bottom displacements during strong submarine earthquake give rise to intensive lowfrequency elastic oscillations of water layer. Nonlinear energy transfer from the elastic oscillations to long gravitational waves may provide an additional contribution to tsunami. The nonlinear tsunami generation mechanism is examined analytically. Finiteness of bottom elasticity is taken into account. General parameters responsible for amplitude and energy of the nonlinear contribution to tsunami wave are revealed.
\end{abstract}

\section{Introduction}

Most tsunamis spring from deformations of ocean bottom during strong submarine earthquakes. Co-seismic bottom motions also give rise to low-frequency elastic oscillations of the water column (Nosov, 1999, 2000). In case of a horizontal bottom, the oscillations are characterized by a discrete set of normal frequencies: $v_{j}=c(1+2 j) / 4 H$, where $j=0,1,2, \ldots, c$ is the sound speed in water, $H$ is the oceanic depth. Typical value of the normal frequency is $v_{0} \sim 0.1 \mathrm{~Hz}$ ( $H=4000 \mathrm{~m}, c=1500 \mathrm{~m} / \mathrm{s}$ ). A clear manifestation of the lowfrequency elastic oscillations has been recently detected in bottom pressure records (Nosov et al., 2005, 2007).

Owing to the existence of the cut-off frequency (Tolstoy and Clay, 1987), the lowest mode $v_{0}=c / 4 H$ formed at a given depth $H$ does not propagate upslope. Therefore, the mode is trapped by localized depressions of bottom, i.e. trenches or holes. Being manifested only at sufficiently large depths (in the open ocean) the low-frequency elastic oscillations are not observed near the shore. However, strong oscillations can contribute to the tsunami by means of nonlin-

Correspondence to: M. A. Nosov

(nosov@phys.msu.ru) ear mechanisms (Novikova and Ostrovsky, 1982; Nosov and Skachko, 2001; Nosov and Kolesov, 2005).

Let us compare the values of the energy of elastic oscillations and surface gravity waves (tsunami) generated by a vertical deformation of a flat bottom. Let the section of the bottom of the area $S(\sqrt{S} \gg H)$ begin to move with a constant velocity $U$ at a given moment of time. The motion lasts during the time $T(T \ll \sqrt{S / g H})$, and then the bottom stops. The permanent displacement height is $U T$. The energy of the tsunami is estimated as the potential energy of the initial elevation of water surface $W_{1}=0.5 \rho g S(U T)^{2}$, where $g$ is the acceleration of gravity and $\rho$ is the water density.

In the frameworks of the model of a compressible fluid, the power transmitted to the water layer by a surface of the movable bottom is determined from the following simple formula (Landau, Lifshitz, 1987): $W_{2}=\rho c U^{2} S T$.

The ratio of the values takes the following form: $W_{2} / W_{1}=2 c / g T$. The bottom rise time T for M8 earthquake obtained from empirical law (see e.g. Kanamori and Anderson, 1975) is $\sim 6$ s, i.e. $W_{2} / W_{1} \approx 50$. It is seen that an essential amount of energy that is transmitted from the moving bottom to the water layer exists in the form of elastic oscillations. Therefore, elastic oscillations are potentially capable to contribute tsunami waves by means of nonlinearity.

Finiteness of bottom elasticity leads to a gradual damping of the elastic oscillations due to refraction of the hydroacoustic waves in the bottom. The exponential decay time for energy (normal incidence) can be estimated as follows (Nosov, 2000):

$\tau=D \frac{H}{c}, \quad D=\frac{\left(1+\rho_{b} V_{p} / \rho c\right)^{2}}{2 \rho_{b} V_{p} / \rho c}$,

where $D \approx 7-9.5$ (Nosov et al., 2007), $V_{p}$ is P-wave velocity, $\rho_{b}$ is bottom density. It is worth reminding that the decay time for amplitude is two times longer. For deep water, the elastic oscillations of water layer can be observed at least during a few minutes after bottom earthquake.

Published by Copernicus Publications on behalf of the European Geosciences Union. 


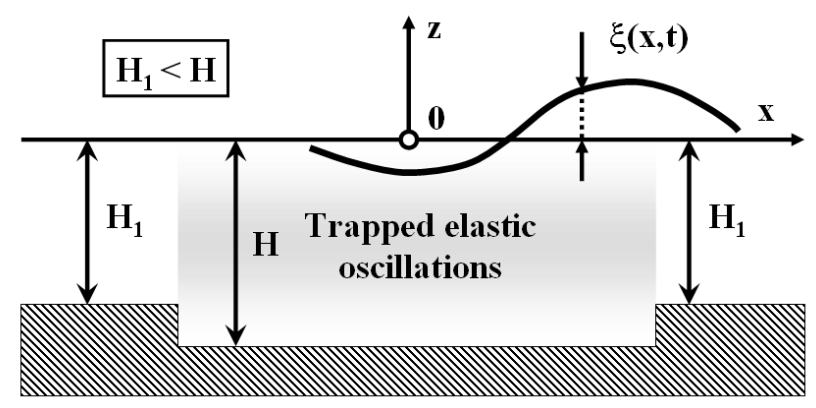

Fig. 1. Mathematical statement of the problem.

The problem of tsunami generation due to nonlinear energy transfer from elastic or forced oscillations of water column to the long gravitational waves has been already analyzed numerically for absolutely rigid bottom (Nosov and Skachko, 2001; Nosov and Kolesov, 2005). Neglecting of the bottom elasticity certainly leads to an overestimation of the nonlinear contribution in tsunami. In the present study, we consider compressible water column lying on an elastic bottom. The problem of tsunami generation by means of nonlinear phenomena is examined analytically.

\section{Nonlinear tsunami source: mathematical model}

Let us consider a layer of an ideal compressible homogeneous fluid in the field of gravity. The mathematical model is based on the non-linear hydrodynamic equations

$$
\begin{aligned}
& \frac{\partial \boldsymbol{v}}{\partial t}+(\boldsymbol{v}, \nabla) \boldsymbol{v}=-\frac{\nabla p}{\rho}+\boldsymbol{g} \\
& \frac{\partial \rho}{\partial t}+\operatorname{div}(\rho \boldsymbol{v})=0 .
\end{aligned}
$$

It is assumed that flow consists of oscillating and timeaveraged components

$\boldsymbol{v}=\langle\boldsymbol{v}\rangle+\boldsymbol{v}, \quad p=\langle p\rangle+p^{\prime}, \quad \rho=\langle\rho\rangle+\rho^{\prime}$,

where $\boldsymbol{v}$ is fluid velocity vector, $\mathrm{p}$ is pressure, $\rho$ is density. Let us specify the averaging interval to be equal to the maximum period of elastic oscillations $4 H_{\max } / c$, where $H_{\max }$ is the maximum depth. Thus, the time-averaged flow can be considered as incompressible fluid motion. Substituting expressions (4) into Eqs. (2) and (3) and averaging these equations in time we obtained

$\frac{\partial\langle\boldsymbol{v}\rangle}{\partial t}=-\frac{\nabla\langle p\rangle}{\langle\rho\rangle}+g+\boldsymbol{\Phi}$,

$\operatorname{div}(\langle\boldsymbol{v}\rangle)=s$,

$\boldsymbol{\Phi}=-\langle(\boldsymbol{v}, \boldsymbol{\nabla}) \boldsymbol{v}\rangle+0.5\left\langle\nabla p^{\prime 2}\right\rangle c^{-2}\langle\rho\rangle^{-2}$

$s=-c^{-2}\langle\rho\rangle^{-1} \operatorname{div}\left\langle p^{\prime} \boldsymbol{v}^{\prime}\right\rangle$,
When deriving Eqs. (5) and (6) we also neglected the nonlinear term $(\langle\boldsymbol{v}\rangle \nabla)\langle\boldsymbol{v}\rangle$ and used the relation $p^{\prime}=c^{2} \rho^{\prime}$.

The non-linearity of Eqs. (2) and (3) introduces additional terms in the time-averaged flow equations (5) and (6). These additional terms $\boldsymbol{\Phi}$ and $s$ can be interpreted as external mass force and distributed mass source; we consider them as a non-linear tsunami source.

In what follows we restrict our approach to a 2-D (plane) problem. The origin of the Cartesian coordinate system Oxz is located at the unperturbed free water surface, and the Ozaxis is oriented vertically upward (Fig. 1).

Generation of gravitational waves by joint action of the external mass force $\boldsymbol{\Phi}=\left(\Phi_{x}, \Phi_{z}\right)$ and the distributed mass source $s$ is described in the framework of the long-wave linear theory. Neglecting vertical acceleration and integrating the Eqs. (5) and (6) along the vertical coordinate from bottom up to free surface, we get the wave equation in terms of free surface displacements $\xi$ :

$\frac{\partial^{2} \xi}{\partial x^{2}}-\frac{1}{g H} \frac{\partial^{2} \xi}{\partial t^{2}}=\frac{1}{g H} Q(x, t)$,

where

$Q(x, t)=\int_{-H}^{0} d z\left[\frac{\partial \Phi_{x}}{\partial x}+\int_{z}^{0} \frac{\partial^{2} \Phi_{z}}{\partial x^{2}} d z^{*}-\frac{\partial s}{\partial t}\right]$.

Compressible fluid layer bounded above and below by a free surface and absolutely rigid bottom respectively is a wave guide. According to the theory of wave guides, elastic oscillations of a semi-bounded domain have a continuous frequency spectrum and any elastic motion can be expressed as a superposition of normal waves (Brekhovskikh and Goncharov, 1994)

$F(x, z, t)=\sum_{j} \int d \omega F_{j}^{0}(\omega) \cos \left(\omega t-m_{j} x\right) \sin \left(n_{j} z\right)$,

$m_{j}=\left(\omega^{2} / c^{2}-n_{j}^{2}\right)^{1 / 2}, \quad n_{j}=\frac{\pi}{2 H}(1+2 j)$

where $m_{j}$ is the horizontal component of the wave vector, and $n_{j}$ is its vertical component. According to dispersion relation (12), only a finite number of modes of a fixed frequency $\omega$ can propagate in horizontal direction. These are called the "propagating modes". For certain modes $m_{j}$ is imaginary. Such modes decrease exponentially and thus can not propagate along the axis $\mathrm{Ox}$.

Let us express functions $v$ and $p^{\prime}$ in terms of the fluid velocity potential

$\boldsymbol{v}=\nabla F, \quad p^{\prime}=-\rho \frac{\partial F}{\partial t}$. 
Considering formulas (7), (8), (11), and (13), we obtain

$\Phi_{x}=\sum_{j k} \int d \omega_{1} \int d \omega_{2} F_{j}^{0}\left(\omega_{1}\right) F_{k}^{0}\left(\omega_{2}\right) m_{2 k} \times$

$\times\left\{m_{1 j} m_{2 k} \sin \left(\omega_{1} t-m_{1 j} x\right) \cos \left(\omega_{2} t-m_{2 k} x\right) \sin \left(n_{j} z\right) \sin \left(n_{k} z\right)-\right.$

$-n_{j} n_{k} \cos \left(\omega_{1} t-m_{1 j} x\right) \sin \left(\omega_{2} t-m_{2 k} x\right) \cos \left(n_{j} z\right) \cos \left(n_{k} z\right)-$

$\left.-c^{-2} \omega_{1} \omega_{2} \sin \left(\omega_{1} t-m_{1 j} x\right) \cos \left(\omega_{2} t-m_{2 k} x\right) \sin \left(n_{j} z\right) \sin \left(n_{k} z\right)\right\}$,

$\Phi_{z}=\sum_{j k} \int d \omega_{1} \int d \omega_{2} F_{j}^{0}\left(\omega_{1}\right) F_{k}^{0}\left(\omega_{2}\right) n_{k} \times$

$\times\left\{-m_{1 j} m_{2 k} \sin \left(\omega_{1} t-m_{1 j} x\right) \sin \left(\omega_{2} t-m_{2 k} x\right) \sin \left(n_{j} z\right) \cos \left(n_{k} z\right)+\right.$

$+n_{j} n_{k} \cos \left(\omega_{1} t-m_{1 j} x\right) \cos \left(\omega_{2} t-m_{2 k} x\right) \cos \left(n_{j} z\right) \sin \left(n_{k} z\right)+$

$\left.+c^{-2} \omega_{1} \omega_{2} \sin \left(\omega_{1} t-m_{1 j} x\right) \sin \left(\omega_{2} t-m_{2 k} x\right) \sin \left(n_{j} z\right) \cos \left(n_{k} z\right)\right\}$,

$s=\frac{1}{c^{2}} \sum_{j k} \int d \omega_{1} \int d \omega_{2} F_{j}^{0}\left(\omega_{1}\right) F_{k}^{0}\left(\omega_{2}\right) \omega_{1} \times$

$\times\left\{m_{1 j} m_{2 k} \cos \left(\omega_{1} t-m_{1 j} x\right) \sin \left(\omega_{2} t-m_{2 k} x\right) \sin \left(n_{j} z\right) \sin \left(n_{k} z\right)+\right.$

$+m_{2 k}^{2} \sin \left(\omega_{1} t-m_{1 j} x\right) \cos \left(\omega_{2} t-m_{2 k} x\right) \sin \left(n_{j} z\right) \sin \left(n_{k} z\right)-$

$-n_{j} n_{k} \sin \left(\omega_{1} t-m_{1 j} x\right) \cos \left(\omega_{2} t-m_{2 k} x\right) \cos \left(n_{j} z\right) \cos \left(n_{k} z\right)+$

$\left.+n_{j}^{2} \sin \left(\omega_{1} t-m_{j} x\right) \cos \left(\omega_{2} t-m_{2 k} x\right) \sin \left(n_{j} z\right) \sin \left(n_{k} z\right)\right\}$.

It is important to stress here that elastic waves, with frequencies smaller than a minimum critical frequency do not exist, i.e. $\omega_{1}>\omega_{0}$ and $\omega_{2}>\omega_{0}$. Replacing in expressions (14)-(16) the products of "sin" and "cos" with sums of trigonometric functions, we get terms that oscillate with frequencies $\omega_{1}+\omega_{2}$ and $\omega_{1}-\omega_{2}$. While averaging the expressions in time, the terms with frequencies $\omega_{1}+\omega_{2}$ vanish. At the same time, the terms with frequencies $\omega_{1}-\omega_{2}$ provide a nonzero contribution, but only if $\left|\omega_{1}-\omega_{2}\right|<\omega_{0}$. Thus the domain of integration (Fig. 2) is a narrow band about the line $\omega_{1}=\omega_{2}$.

According to Eq. (10), the following three values contribute to the generation of long gravitational waves:

$$
\begin{aligned}
\text { "X" } & =\int_{-H}^{0} \frac{\partial \Phi_{x}}{\partial x} d z \\
“ Z " & =\int_{-H}^{0} \int_{z}^{0} \frac{\partial^{2} \Phi_{z}}{\partial x^{2}} d z^{*} d z, \\
" S " & =-\int_{-H}^{0} \frac{\partial s}{\partial t} d z .
\end{aligned}
$$

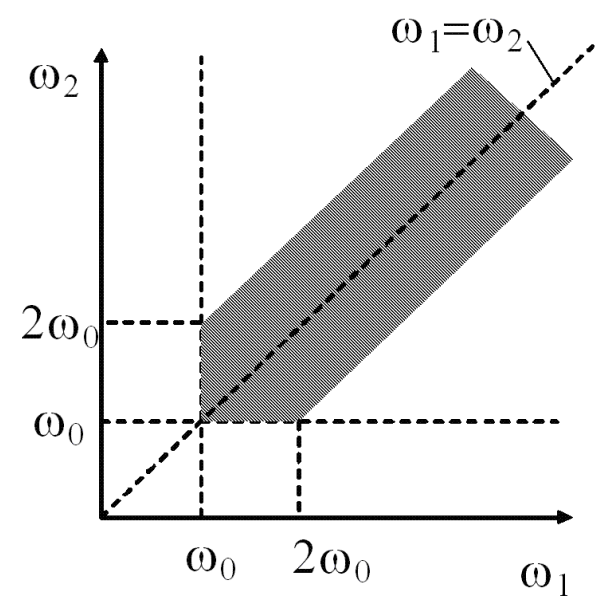

Fig. 2. The domain of integration.

Omitting intermediate steps, we get the final expressions these three values:

$$
\begin{aligned}
“ X " & =\frac{H}{8 c^{4}} \sum_{j} \int d \omega_{1} \int d \omega_{2} F_{j}^{0}\left(\omega_{1}\right) F_{j}^{0}\left(\omega_{2}\right) \\
& \cos \left[\left(\omega_{1}-\omega_{2}\right) t-\left(m_{1 j}-m_{2 j}\right) x\right] \omega \Delta \omega^{3} \frac{n^{2}}{m^{2}} \\
& =\frac{H}{4 c^{4}} \sum_{j} \int d \omega_{1} \int d \omega_{2} F_{j}^{0}\left(\omega_{1}\right) F_{j}^{0}\left(\omega_{2}\right) \\
& \cos \left[\left(\omega_{1}-\omega_{2}\right) t-\left(m_{1 j}-m_{2 j}\right) x\right] \omega^{2} \Delta \omega^{2} \frac{n^{2}}{m^{2}}+ \\
& +\frac{H}{8} \sum_{j \neq k} \int d \omega_{1} \int d \omega_{2} F_{j}^{0}\left(\omega_{1}\right) F_{k}^{0}\left(\omega_{2}\right) \\
& \cos \left[\left(\omega_{1}-\omega_{2}\right) t-\left(m_{1 j}-m_{2 k}\right) x\right] \times\left(m_{1 j}-m_{2 k}\right)^{2} \\
& \left\{\left(\frac{\omega_{1} \omega_{2}}{c^{2}}-m_{1 j} m_{2 k}\right)\left(\frac{1+2 k}{j-k}+\frac{1+2 k}{1+j+k}\right)\right. \\
& \left.-n_{j} n_{k}\left(\frac{1+2 k}{j-k}-\frac{1+2 k}{1+j+k}\right)\right\}
\end{aligned}
$$

$$
\begin{array}{r}
" S "=-\frac{H}{4 c^{4}} \sum_{j} \int d \omega_{1} \int d \omega_{2} F_{j}^{0}\left(\omega_{1}\right) F_{j}^{0}\left(\omega_{2}\right) \\
\cos \left[\left(\omega_{1}-\omega_{2}\right) t-\left(m_{1 j}-m_{2 j}\right) x\right] \omega^{2} \Delta \omega^{2} .
\end{array}
$$




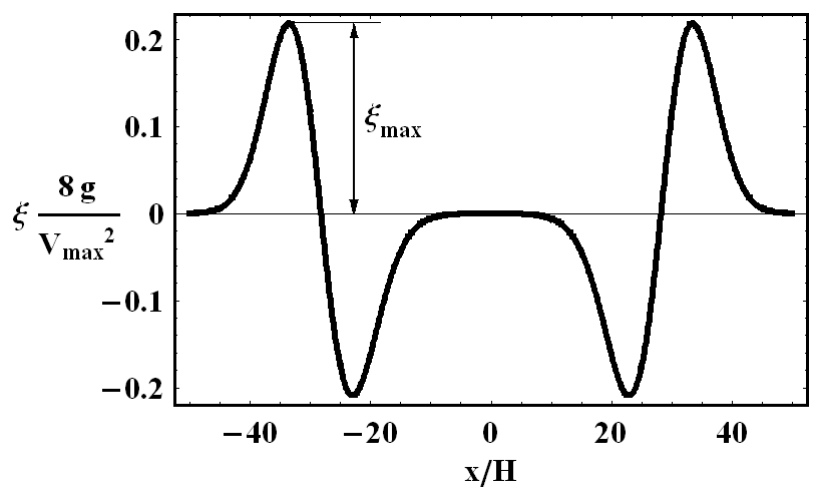

Fig. 3. Typical wave profile generated by the nonlinear tsunami source. Calculations performed at $L=10 H, \tau=2 \sqrt{H / g}$, $t=3 L / \sqrt{g H}$.

When deriving the expressions (18)-(20), the following formulas and assumptions were used:

$\int_{-H}^{0} \cos \left[\frac{\pi \beta}{H} z\right] d z=H \frac{\sin [\pi \beta]}{\pi \beta}=\left\{\begin{array}{l}H, \beta=0 \\ 0, \beta= \pm 1, \pm 2, \pm 3, \ldots\end{array}\right.$,

$\int_{-H}^{0} d z \int_{z}^{0} d z^{*} \sin \left[\frac{\pi \beta}{H} z^{*}\right]=\frac{H^{2}}{\pi^{2} \beta^{2}}[\sin (\pi \beta)-\pi \beta]=\left\{\begin{array}{l}0, \beta=0 \\ -H^{2} \pi^{-1} \beta^{-1}, \\ \beta= \pm 1, \pm 2, \pm 3, \ldots\end{array}\right.$,

$\beta=j-k$ or $\beta=1+j+k, \Delta m=m_{1}-m_{2}, \Delta \omega=\omega_{1}-\omega_{2}$,

$\Delta m \approx \frac{\omega}{c^{2} m} \Delta \omega-\frac{1}{2 c^{2} m} \frac{n^{2}}{m^{2}} \Delta \omega^{2}$.

Comparison of expressions (18)-(20) allow us to conclude that the vertical component of the force $\Phi_{z}$ always dominates in the generation of long waves, whereas the contributions of the horizontal component $\Phi_{x}$ and the distributed mass source $s$ are negligible (" $X$ " $/ “ Z$ " $\sim \Delta \omega / \omega \ll 1$, " $S$ " / $Z$ " $\left.\sim m^{2} / n^{2} \ll 1\right)$. This conclusion is also confirmed by results of our numerical simulation.

Neglecting values " $X$ " and " $S$ ", we reduce the Eq. (9) to the following form:

$\frac{\partial^{2} \xi}{\partial x^{2}}-\frac{1}{g H} \frac{\partial^{2} \xi}{\partial t^{2}}=\frac{1}{g H} \frac{\partial^{2}}{\partial x^{2}} \int_{-H}^{0} \int_{z}^{0} \Phi_{z} d z^{*} d z$

In order to estimate function $\Phi_{z}$ analytically, we consider only the lowest mode $v_{0}=c / 4 H$ which is trapped by a localized depression. We assume the step-like bottom profile $\left(H_{1}<H, H-H_{1} \ll H\right)$ which is shown on Fig. 1 . The fluid velocity potential for the trapped mode is expressed as follows:

$F(x, z, t)=F_{0} \cos \left(\frac{c \pi}{2 H} t\right) \sin \left(\frac{\pi}{2 H} z\right)$ where $F_{0}$ is amplitude of the lowest mode. Substituting expression (22) in formula (7) we obtain

$\Phi_{z}=\frac{F_{0}^{2} \pi^{3}}{16 H^{3}} \sin \left(\frac{\pi z}{H}\right), \int_{-H}^{0} \int_{z}^{0} \Phi_{z} d z^{*} d z=-\frac{F_{0}^{2} \pi^{2}}{16 H}$

When deriving (23) we neglect the horizontal derivatives in comparison with vertical derivatives. It is worth noting that actually we assume the amplitude $F_{0}$ being a weak function of coordinate $\mathrm{x}$.

Due to bottom elasticity, the energy of trapped elastic oscillations decreases exponentially, thus the amplitude $F_{0}$ decreases in time. The exponential dacay time for the amplitude is $2 \tau$ (Eq. 1). Let us specify the following spatio-temporal law for the amplitude: $F_{0}(x, t)=F_{0}^{\max } \exp \left[-t / 2 \tau-(x / L)^{2}\right]$, where $L$ is a characteristic length of tsunami source $(L \gg H), F_{0}^{\max }$ is the maximum mode amplitude which can be expressed via maximum mass velocity of fluid $F_{0}^{\max }=V_{\max } 2 H / \pi$. The value $V_{\max }$ also can be interpreted as the maximum bottom velocity of vertical bottom displacement.

The analytical solution of the heterogeneous wave Eq. (9) is given by well-known integral formula. Introducing dimensionless variables $(x / H, t \sqrt{g / H})$ and omitting intermediate transformations, we obtain

$\xi(x, t)=\frac{V_{\max }^{2}}{8 g} \int_{0}^{t} d t^{*}\left(\left.\frac{\partial \varphi}{\partial x}\right|_{\left[x+\left(t-t^{*}\right), t^{*}\right]}-\left.\frac{\partial \varphi}{\partial x}\right|_{\left[x-\left(t-t^{*}\right), t^{*}\right]}\right)$,

where $\varphi(x, t)=\exp \left[-t / \tau-2(x / L)^{2}\right]$.

\section{Discussion of results}

A typical gravitational wave, formed by the non-linear source, is presented on Fig. 3. This wave profile was calculated from Eq. (24). The profile consists of a positive leading crest and a virtually symmetric negative tail. The wave length corresponds to the horizontal size of the source (i.e. $\sim L$ ). It is seen from Eq. (24) that the amplitude of the wave is mostly determined by the factor $V_{\max }^{2} / 8 \mathrm{~g}$. Thus, a noticeable contribution to tsunami amplitude can be expected if $V_{\max }>\sim 10 \mathrm{~m} / \mathrm{s}$. Such great values of the mass velocity may result either from very rapid co-seismic bottom displacements or from a resonant pumping of energy in the elastic oscillations of water layer. The resonance may occur if the normal frequency $v_{i}$ (e.g. $\left.v_{0}\right)$ fits the spectrum of seismic bottom trembling.

The wave amplitude $\xi_{\max }$ and energy $E=\rho g \int_{-\infty}^{+\infty} \xi^{2} d x$ are plotted on Fig. 4 as functions of the exponential decay time $\tau$ (see Eq. 1). The plots are shown in dimensionless form. Either the amplitude or the energy slightly increases as the value of $\tau$ goes up. According to Eq. (1) the parameter 

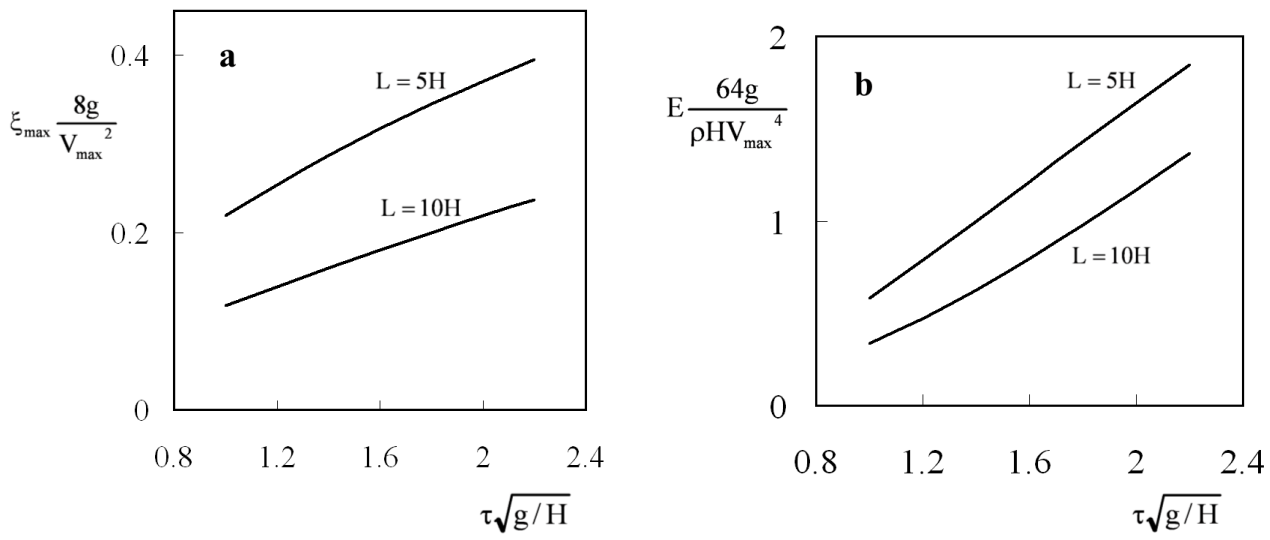

Fig. 4. Wave amplitude (a) and energy (b) as a function of the exponential decay time.

$\tau \sqrt{g / H}$ varies within the range $0-2.1$. We do not consider small values of the parameter. The small values stand for shallow water where manifestations of the elastic oscillations are depressed. The exponential decay time $\tau$ has a simple physical meaning in the problem under consideration. It stands for characteristic duration of acting of the nonlinear tsunami source. In case of absolutely rigid bottom, energy of the elastic oscillations attenuates rather slowly, only because a radiation of hydroacoustic waves in horizontal direction. Finiteness of bottom elasticity leads to a more effective decay of elastic oscillations due to refraction of hydroacoustic waves in bottom. This is why our previous estimations performed for absolutely rigid bottom (e.g. Nosov and Kolesov, 2005) overestimate contribution of nonlinearity in tsunami wave.

It is also seen from Fig. 4 that the source characteristic length $L$ has an influence on the amplitude and the energy. Actually this influence results from changing of spatial steepness of the function $\varphi(x, t)$ from Eq. (24). Assuming another kind of spatial distribution, we surely obtain slightly different values of the amplitude and energy. Anyway, concrete definition of function $\varphi(x, t)$ is necessary to solve the problem analytically.

In conclusion, it is important to note that the non-linear mechanism can lead to observable, though not dominant contribution to the tsunami amplitude. However, the non-linear effects can play a dominant role during quick bottom motions without residual displacements, when the traditional linear mechanism is not able to effectively generate gravitational waves.

We did not consider here contribution of the modes $j>0$, they may also provide an additional contribution in tsunami. We choose just exponentially decaying time-function for the source, whereas, in reality there is a finite time of energy pumping to the elastic oscillations. At last, we examined the case of flat horizontal bottom only and did not consider a realistic bottom profile. All the listed points are subjects for further numerical modeling of the problem.
Acknowledgements. This work was supported by the Russian Foundation for Basic Research, project 07-05-00414 and the Federal Program for the Support of Leading Scientific Schools, project no. NSh-2104.2003.5.

Edited by: P. Fabian

Reviewed by: two anonymous referees

\section{References}

Brekhovskikh, L. M. and Goncharov, V. V.: Mechanics of Continua and Wave Dynamics, 2nd ed., Berlin; New York, SpringerVerlag, 1994.

Kanamori, H. and Anderson, D. L.: Theoretical basis of some empirical relations in seismology, Bull. Seism. Soc. Am. 65, 10731095, 1975.

Landau, L. D. and Lifshitz, E. M.: Fluid Mechanics, 2nd ed., Oxford, Pergamon, 1987.

Nosov, M. A. and Kolesov, S. V.: Nonlinear tsunami generation mechanism in compressible ocean, Vestnik Moskovskogo Universita, Ser. 3 Fizika Astronomiya, 3, 51-54, 2005.

Nosov, M. A. and Skachko, S. N.: Nonlinear tsunami generation mechanism, Nat. Hazards Earth Syst. Sci., 1, 251-253, 2001, http://www.nat-hazards-earth-syst-sci.net/1/251/2001/.

Nosov, M. A. and Kolesov, S. V.: Elastic oscillations of water column in the 2003 Tokachi-oki tsunami source: in-situ measurements and 3-D numerical modelling, Nat. Hazards Earth Syst. Sci., 7, 243-249, 2007, http://www.nat-hazards-earth-syst-sci.net/7/243/2007/.

Nosov, M. A., Kolesov, S. V., Ostroukhova, A. V., Alekseev, A. B., and Levin, B. W.: Elastic oscillations of the water layer in a tsunami source, Doklady Earth Sci., 404(7), 1097-1100, 2005.

Nosov, M. A.: Tsunami generation in a compressible ocean by vertical bottom motions, Izvestiya, Atmos. Ocean Phys., 36(5), 661-669, 2000.

Nosov, M. A.: Tsunami generation in compressible ocean, Physics and Chemistry of the Earth, Part B: Hydrology, Oceans and Atmosphere, 24(5), 437-441, 1999.

Novikova, L. E. and Ostrovsky, L. A.: On an acoustic mechanism of tsunami wave generation, Oceanology, 22(5), 693-697, 1982. 
Tolstoy, I. and Clay, C. S.: Ocean Acoustics - Theory and Experiment in Underwater Sound, 2nd ed., American Institute of Physics, New York, 1987. 